\title{
Ischaemic stroke subtypes had different short term and long term functional outcomes, mortality, and recurrence rates
}

\author{
Petty GW,Brown RD Jr, Whisnant JP, et al. Ischemic stroke subtypes. A population-based study of functional outcome, \\ survival, and recurrence. Stroke 2000 May;31:1062-8.
}

QUESTION: In patients who have had a first ischaemic stroke, are short term and long term functional outcomes, mortality rates, and recurrence rates different for subtypes of stroke (large vessel cervical or intracranial atherosclerosis with stenosis, cardioembolic, lacunar, and unknown cause)?

Design

Inception cohort followed for up to 5 years (mean $3.2 \mathrm{y}$ ).

Setting

A community study in Rochester, Minnesota, USA.

\section{Patients}

454 patients with a first ischaemic stroke from 1985 to 1989. \{Mean patient age was 72 years for atherosclerotic stroke, 80 for cardioembolic stroke, 73 for lacunar stroke, and 76 for stroke of unknown cause. Women had $32 \%$ of the atherosclerotic strokes, $67 \%$ of the cardioembolic strokes, $57 \%$ of the lacunar strokes, and $67 \%$ of the strokes of unknown cause. $\}^{*}$

\section{Assessment of prognostic factors}

Stroke subtype, severity, age, sex, and predefined risk factors \{previous transient ischaemic attack, hypertension, diabetes, smoking, congestive heart failure, myocardial infarction, angina, mitral valve disease, and atrial fibrillation $\}^{*}$.

\section{Main outcome measures}

Functional outcome using the Rankin disability score (from grade 1 [no significant disability] to grade 5 [severe disability, bedridden, incontinent, and requiring constant nursing care and attention]), mortality, and recurrent stroke.

Sources of funding: National Institutes of Health and Agency for Health Care Policy Research.

For correspondence: Dr G W Petty, Division of Cerebrovascular Diseases, Mayo Clinic, 200 First Street

Southwest, Rochester,

MN 55905, USA. Fax $+15072664419$

Outcomes for patients with a first ischaemic stroke by subtype

\begin{tabular}{lllll} 
Outcomes & Atherosclerotic & Cardioembolic & Lacunar & $\begin{array}{l}\text { Unknown } \\
\text { cause }\end{array}$ \\
Mortality at 30 days & $8.1 \%$ & $30.3 \%$ & $1.4 \%$ & $14.0 \% \dagger$ \\
\hline Mortality at 5 years & $32.2 \%$ & $80.4 \%$ & $35.1 \%$ & $48.6 \%$ \\
\hline Recurrent stroke at 30 days & $18.5 \%$ & $5.3 \%$ & $1.4 \%$ & $3.3 \%$ \\
\hline Recurrent stroke at 5 years & $40.2 \%$ & $31.7 \%$ & $24.8 \%$ & $33.2 \% \dagger$ \\
\hline Good function at 1 year & $53.4 \%$ & $26.7 \%$ & $81.9 \%$ & $50.3 \%$ \\
\hline
\end{tabular}

†Not significant; all other categories statistically different across subtype of stroke.

\section{Conclusion}

Patients had varying rates of function, mortality, and recurrence depending on the subtype of ischaemic stroke.

* Petty GW, Brown RD, Whisnant JP, et al. Ischemic stroke subtypes: a population-based study of incidence and risk factors. Stroke 1999;30:2513-6.

\section{COMMENTARY}

The study by Petty et al confirms what other studies have shown: patients with small vessel strokes generally function well, and patients with large vessel strokes, either from atherosclerosis or cardiac embolism, do not. What is surprising about the study is the high 30 day recurrence rates $(18 \%)$ for patients with atherosclerosis, a rate higher than that in other prospective studies, although the registries used in the studies were not population based. ${ }^{1}$

The early stroke recurrence data need to be confirmed in other studies. If they are confirmed, what are possible explanations of this high rate? The authors point toward iatrogenesis as a potentially preventable cause of early stroke; 4 of the 13 strokes in the atherosclerosis group were related to iatrogenesis. This finding stresses the need for hospitals and physicians to track and study their own complication rates to reduce medical error and improve patient safety. A residual non-iatrogenic increased risk for early recurrence in the atherosclerosis group still exists and needs to be reduced. In addition to the increased rate of early recurrence, patients with atherosclerotic stroke also had a higher long term rate of stroke recurrence, a finding that reinforces the need to promptly evaluate patients, identify the most likely stroke mechanism, and provide targeted treatment.

Finally, the investigators have good data to develop a patient outcome prediction model based on stroke subtype, age, and sex that could be useful for clinicians and patients. Judith A Hinchey, MD

Robert G Holloway, MD, MPH University of Rochester Rochester, New York, USA

1 Sacco RL, Foulkes MA, Mohr JP, et al. Determinants of early recurrence of cerebral infarction: the stroke data bank. Stroke 1989;20:983-9.

2 Moroney JT, Bagcita E, Paik MC, et al. Risk factors for early recurrence after ischemic stroke: the role of stroke syndrome and subtype. Stroke 1998;29:2118-24. 